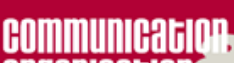
Organlofidin.

\section{Communication et organisation}

Revue scientifique francophone en Communication

organisationnelle

$18 \mid 2000$

Non-verbal, communication, organisation

\title{
Auto-perception des inférences liées au traitement des informations non-verbales sur autrui : le cas de la construction du jugement chez des magistrats
}

\section{Marie-Agnès de Gail et Nicolas Guéguen}

\section{(2) OpenEdition}

Édition électronique

URL : https://journals.openedition.org/communicationorganisation/2415

DOI : 10.4000/communicationorganisation.2415

ISSN : $1775-3546$

Éditeur

Presses universitaires de Bordeaux

Édition imprimée

Date de publication : 1 novembre 2000

ISSN : 1168-5549

Référence électronique

Marie-Agnès de Gail et Nicolas Guéguen, « Auto-perception des inférences liées au traitement des informations non-verbales sur autrui : le cas de la construction du jugement chez des magistrats », Communication et organisation [En ligne], 18 | 2000, mis en ligne le 27 mars 2012, consulté le 05 août 2021. URL : http://journals.openedition.org/communicationorganisation/2415 ; DOI : https://doi.org/ 10.4000/communicationorganisation.2415

Ce document a été généré automatiquement le 5 août 2021

(C) Presses universitaires de Bordeaux 


\title{
Auto-perception des inférences liées au traitement des informations non- verbales sur autrui : le cas de la construction du jugement chez des magistrats
}

\author{
Marie-Agnès de Gail et Nicolas Guéguen
}

\section{Introduction}

1 À propos des descriptions «scientifiques » de la personnalité, Jean-Marc Monteil ${ }^{1}$ qui rassemble des conceptions théoriques des chercheurs en socio-cognition, avance ceci : «La structure de la personnalité d'un individu se situerait, plus probablement, dans la sphère cognitive de celui qui juge, que dans le sujet dont on observe le comportement. »

2 Les recherches sur l'attribution et plus particulièrement sur l'auto-attribution ont montré que nous n'avons pas accès aux processus mentaux qui déterminent la plupart de nos jugements, de nos comportements et même de nos affects. Un mécanisme d'inférence sur son propre état permet à l'individu de se décrire lui-même, d'analyser sa bonne humeur ou son agressivité en fonction de « signes extérieurs ». C'est grâce aux images que la personne a d'elle-même, qu'elle va décoder les comportements des autres. "L'attribution interne de causes impersonnelles et personnelles sont des faits quotidiens qui déterminent une grande partie de notre compréhension et de nos réactions à l'environnement " (Jean-Léon Beauvois, 1984). Qu'il s'agisse d'interpréter nos propres motivations ou celles d'autrui. c'est à partir d'éléments extérieurs « signifiants » que nous allons mobiliser une « théorie » cohérente de la réalité.

3 En outre, des associations de traits de personnalité ou "taxinomies » qui relient arbitrairement des caractéristiques telles qu'élégance et sociabilité, seraient fortement 
liées à la finalité sociale des pratiques, dépendantes de ce que Henri Paicheler (1984) appelle des schèmes de causalité individuelle et sociale. "L'accès à un type d'emploi, la place occupée dans le système social passe par la possession d'un capital de personnalité ». L'efficacité du processus résiderait dans le fait que si les acteurs sociaux ne « connaissent » pas la théorie, ils l'utilisent et la partagent ensemble au sein de leur groupe culturel de référence. S'ils sont capables d'en énoncer les effets "naturellement ", la logique des mécanismes en œuvre ne saurait leur apparaître de façon aussi évidente.

4 L'image que l'on se fait d'autrui serait déterminée par la présence d'indices tels que l'attitude physique, les vêtements, la conformité aux règles sociales... Dans la construction du jugement, certaines caractéristiques non-verbales se révèlent déterminantes alors que d'autres sont secondaires. Ces associations sémantiques préétablies agiraient à un double niveau : au niveau des descripteurs et des indices qui permettent d'identifier la situation et au niveau de l'image que le juge se fait de sa propre capacité à apprécier objectivement tous les paramètres : si la situation est facile ou non à « décoder », si l'ensemble du dossier est cohérent ou « jugeable ».

\section{Ce que cache le concept de personnalité}

5 Comme le concept de quotient intellectuel et d'attitude, celui de personnalité occupe une place fondamentale dans l'édifice psychologique. Fondamentale à cause de l'importance théorique que les psychologues lui ont attribuée, mais aussi, et on a déjà pu s'en rendre compte, à cause des implications dans la vie courante : la personnalité se jauge notamment en orientation scolaire, en sélection professionnelle et dans les tribunaux ; avec elle on joue son avenir et parfois sa tête. Quelle réalité cache-t-elle en fait? (Jacques Philippe Leyens, 1986).

6 Il existe des modèles qui traitent des différences individuelles que nous mettons en œuvre quand nous décrivons quelqu'un sous la forme de traits de personnalité. Nous apprenons au cours de notre vie un certain nombre de scripts qui nous permettent ensuite de trouver une cohérence aux conduites manifestées par autrui, mais aussi d'orienter la description de son comportement ou de faits apparemment anodins, comme des gestes ou des attitudes manifestées au cours d'un entretien. Tout se passe comme si nous transportions dans notre tête une matrice de corrélation de traits. Selon Solomon Asch (1952), des éléments de base entraîneraient une impression globale qui structurerait ensuite nos perceptions ultérieures, donnant lieu à des inférences systématiques.

7 Renforçant ce processus, nos limites cognitives nous obligent à avoir recours à un processus bien connu des cognitivistes : la catégorisation. Plus un objet, une couleur ou un concept est codifiable plus on se le rappelle aisément. Ces processus simplificateurs nous facilitent le traitement des informations mais il en résulte aussi une altération de notre objectivité. La psychologie cognitive, psychologie du traitement de l'information, montre donc comment l'individu intègre et mémorise les informations en codant les données qu'il reçoit. Ces schémas permettent d'identifier les «stimuli ", de les classer, de les mettre en relation avec d'autres. Le trait de personnalité constituerait donc un schéma conceptuel d'intégration des informations psychologiques. Certains traits s'assemblent « naturellement ", d'autres s'excluent tout aussi simplement, et font ainsi partie des représentations sociales, comme du reste, la plupart des préjugés sociaux. 
L'apparente évidence des relations entre certains traits (cooccurrence) serait socialement prédéterminée et serait implicite, le sujet n'ayant pas conscience de mobiliser un stéréotype. L'on a pu, dans différentes expériences de psychologie sociale, mesurer la typologie et le caractère systématique de ces portraits robots. La perception immédiate d'une personne se baserait sur quelques éléments qui permettrait ensuite de construire un jugement cohérent. Certains critères physiques auraient une incidence vérifiée (Loren Chapman \& Jean Chapman, 1969) : Les épaules larges annonceraient la grosse tête l'intelligence, la taille le masculinité, une statut social... Les critères de beauté, de force ou de prestance physique seraient déterminants, mais d'autres paramètres survenus lors d'un entretien pourraient aussi orienter un jugement, comme le fait de renverser une tasse de café (Richard Nisbett, 1977) ou le contexte "riche " versus « pauvre » de l'évaluation (John Darley, 1983).

\section{« Utilité » des théories implicites de la personnalité (TIP)}

8 Quand nous traitons de l'information psychologique, dans un contexte social finalisé, nous organisons notre connaissance de l'autre de manière orientée, en mobilisant notamment ce que les psychologues sociaux appellent des théories implicites de la personnalité. (Le terme précis de théorie implicite de la personnalité a été introduit par Jérôme Borner et Ronaldo Tagiuri en 1954). Quand un individu en décrit un autre, il mobiliserait une théorie «toute faite» qui fait référence, qui semble être connue de tout le monde semblant aller de soi comme une sorte de bon sens. Mais ces descriptions apparemment anodines seraient largement influencées par des a priori ayant une fonction de rationalisation. Le rôle principal du trait de personnalité serait de rendre stables et cohérents les comportements d'autrui, de justifier notre propre attitude visà-vis des autres, mais aussi de justifier les normes sociales ou organisationnelles.

9 L'utilité des théories implicites de la personnalité serait double, cognitive et sociale: d'une part elles rendent plus lisible l'environnement, « ...elle donne accès à un monde plus simple, plus prévisible et mieux à même d'être expliqué et donc contrôlé...» (Jacques-Philippe Leyens 1986). D'autre part elles permettent d'interpréter cet environnement, de lui donner un sens. Elles légitiment les faits sociaux notamment les valeurs, les croyances, les normes, les idéologies... telle l'idée rémanente, d'origine religieuse et largement confortée par les TIP que chacun ne reçoit que ce qu'il mérite (en fonction de ce qu'il est, des efforts qu'il fait, voire des qualités morales qu'il possède).

10 La fonction de naturalisation sociale qui s'exprime au travers des TIP est extrêmement importante pour notre propos car elle permet de comprendre le mécanisme de légitimisation présent dans les processus d'évaluation sociale (qu'ils soient scolaires, professionnels ou pénaux).

11 Pour Jacques-Philippe Leyens, au niveau des groupes, nations, races, religions, et autres catégories sociales, les stéréotypes ont une fonction importante de justification. Les attentes stéréotypées sont normatives, le fait que l'on n'attende pas le même comportement, le même langage, le même habillement, d'un jeune «beur ", que d'un "jeune homme de bonne famille», est partagé sous la forme d'un modèle explicatif systématisé qui biaise l'analyse des informations. "À la longue, le réel finit par ressembler à nos représentations... Les règles et les contenus de cette pensée 
représentative finissent par constituer autour de nous un véritable environnement dans lequel le physique et le social fusionnent». Ce véritable environnement, dont parle Serge Moscovici (1984), serait bien de nature double, constitué à la fois de faits réels et de représentations, tels les stéréotypes. Entre les deux un rapport étroit se crée, donnant l'illusion d'une seule réalité.

Lorsque l'on demande à des juges d'expliquer la criminalité de personnes issues de milieux favorisés ou défavorisés, l'on s'aperçoit qu'ils n'expliquent pas les choses de la même façon. Dans un cas les causes sont plutôt imputées à des facteurs dispositionnels, dans l'autre cas à des facteurs circonstanciels. Les qualificatifs employés diffèrent notablement, comme si l'origine sociale autorisait à posséder certaines qualités et pas d'autres. La caractéristique attributive du jugement varierait donc en fonction de la position sociale de l'individu jugé. La détermination de l'image de soi à travers ces confrontations intercatégorielles et la justification en miroir, ipso facto, des sanctions requises en cas de déviance contribuerait à créer un ensemble cohérent et stable.

La psychologie socio-cognitive intègre l'expression des processus cognitifs comme outil de construction de la connaissance, mais non comme déterminant cette connaissance (Jean-Léon Beauvois 1984). Ces processus répondent plutôt, selon elle, à une fonction sociale: celle de légitimer les normes, les valeurs et les croyances inhérentes aux sociétés et aux organisations.

\section{Les indices non-verbaux dans la construction du jugement : hypothèses théoriques}

14 Des auteurs tels qu'Henri Paicheler dans son Epistémologie du sens commun montrent comment la perception d'autrui est déterminée par la présence d'indices physiques et notamment par la forme du visage. Dans la formation d'une impression sur autrui, certaines caractéristiques non-verbales se révèlent périphériques alors que d'autres sont plus centrales, elles se corrèlent avec des descripteurs personnologiques associés entre eux sous forme de cohérences sémantiques préétablies entre l'aspect physique et la situation dans laquelle est émis le jugement. On sait notamment qu'il y a un effet de l'attrait physique ${ }^{2}$ sur l'employabilité, les décisions de justices et que l'on prête des caractéristiques personnelles plus favorables et un avenir plus positif aux personnes physiquement attrayantes.

15 Une recherche précédente ${ }^{3}$ nous avait amenée à vérifier que le traitement des indices non-verbaux précède la construction du jugement dans le contexte du travail social et dans le contexte du recrutement professionnel. La relation de communication implicite qui s'établit intuitivement entre l'évaluateur et l'évalué initialise et conditionne le traitement des informations. Ces interactions para-verbales seraient instituées comme chez les animaux selon des codes archaïques qui échappent largement au conscient (règles de déplacement et de distance dans l'espace par exemple). Mais dans les interactions humaines, les capacités métacognitives qui permettent notamment d'avoir un avis sur ses propres inférences compliquent largement les choses. En effet les études sur la jugeabilité ${ }^{4}$ montrent comment des indices de deuxième ordre nous permettent de nous faire une opinion sur le bien-fondé de notre propre jugement. Il suffit de suggérer à quelqu'un qu'il possède les informations ou les compétences nécessaires pour que sa croyance en la pertinence de son avis augmente de façon très significative. 
16 En outre, quand un profil est "cohérent », il est facilement «identifiable », donc «jugeable »le dossier ne requiert pas de complément d'information. Les situations complexes, plus difficiles à évaluer, sont plus contraignantes. Notre hypothèse est qu'en introduisant une surcharge cognitive pour l'évaluateur, les dossiers atypiques induiraient un biais négatif à l'encontre de la personne « jugée », c'est en tous les cas ce que nous avions vérifié dans le contexte du recrutement professionnel. Si les magistrats ont sans doute bien conscience du risque d'être influencé par des stéréotypes sociaux, ils n'ont pas forcément une idée très réaliste du fait que comme tous les évaluateurs "socio-cognitifs », ils détestent les situations atypiques qui ne se réfèrent pas à des schémas d'interprétation classiques. L'incertitude déstabilise, elle est vécue comme une double menace : au niveau individuel car elle produit un état de dissonance, au niveau collectif car les experts garantissent la cohésion sociale, pour être efficients ils ne doivent pas douter. Ce processus s'appréhenderait dans de nombreux contextes organisationnels (justice, éducation, travail social, gestion des ressources humaines, recherche...). Les spécialistes de l'évaluation d'autrui sont investis par leurs organisations d'un statut d'expert, donc d'une capacité à juger.

17 L'incohérence d'un sujet produirait un effet négatif sur le jugement car elle est inacceptable en l'état, psychologiquement et socialement. Ce ne sont pas les indices non-verbaux qui, dans un tribunal, provoquerait un effet positif ou négatif sur le jugement. Ce serait plutôt la congruence de l'ensemble des éléments entre eux qui conférerait une dimension évaluative plus ou moins favorable au prévenu.

\section{La construction du jugement chez des magistrats : extraits d'entretiens}

(Le compte rendu complet des entretiens a été distribué lors de la communication)

19 Aujourd'hui nous souhaitons illustrer nos propos en livrant ici la synthèse d'entretiens réalisés auprès de jeunes magistrats. Ceux-ci, durant leur formation, ont été partiellement instruits de ces problématiques, comment en tiennent-ils compte dans leurs pratiques et dans leurs discours?

20 Comment justifient-ils le bien fondé de leurs conclusions sur la personnalité des individus qu'ils évaluent? Quelle perception ont-ils de l'incidence du mode relationnel qui s'installe durant les interrogatoires ou les audiences de cabinet?

21 Il s'agit donc d'étudier bien $\mathrm{F}$ auto-perception des inférences réalisées par les magistrats eux-mêmes et non pas de recenser et de décrire les contenus de ces inférences.

22 Notre méthode n'est pas expérimentale : nous nous basons sur les déclarations des magistrats que nous interviewons. Même si nous utilisons l'entretien non-directif, qui permet d'aller au-delà des intentions conscientes de la personne interrogée, la plupart des informations qui nous sont livrées sont déjà « rationalisées ».

Toutes les phrases en italiques sont extraites des interviews. 


\section{La personnalité comme facteur prédisposant :}

"La notion de facteur prédisposant est très importante en droit pénal, ce peut être l'origine sociale (défavorisée), la conjoncture (divorce, chômage) mais aussi la personnalité (instable, fragile...)».

\section{Cohérence entre les différentes informations du dossier}

"Chaque élément (ait partie d'un faisceau, ça colle ou ça ne colle pas avec le personnage, 1 ensemble doit être cohérent"

\section{Une relation précède et prépare le jugement}

Dans les interrogatoires préliminaires du juge, un mode de relation s'installe avec le prévenu qui aura une incidence sur le déroulement de l'instruction.

« Des liens se créent dans les entretiens préparatoires ».

\section{Existe-t-il des schémas types?}

Même si d'après les magistrats interrogés les schémas types n'existent pas et qu'ils savent ne pas se fier à leur "première impression", il y aurait d'après eux des catégories caractéristiques qui ne doivent évidement pas être systématisées mais qui semble « aller avec le reste».

"Les pères incestueux, plutôt chétifs, un peu voûtés qui ont le regard fuyant »(les délinquants sexuels en général auraient souvent ce profil)

\section{La question de l'attrait physique}

À aucun moment il n'a été possible d'aborder directement la question de l'attrait physique comme si cette question n'était pas acceptable dans ce contexte. La seule évocation de cette variable fut quand un magistrat expliqua qu'il avait été la cible de tentatives « grossières » de séduction.

\section{Expertise et bien fondé du jugement}

«Il y a de moins en moins de schémas types "

«Dans les cas difficiles on passe plus de temps »

«D'une manière générale les magistrats sont des gens consciencieux »

\section{Conclusion}

« Il y a donc selon nous deux dimensions de la situation de jugement: d'une part des éléments informants utilisables pour réaliser un jugement et d'autre part des éléments de définition de la situation, des éléments susceptibles de la définir ou non comme une situation correspondant au « script catégoriel » des situations où un jugement peut être formulé. De telles «indications méta-informationnelles peuvent donner à un observateur l'impression qu'il est - ou qu'il n'est pas - «objectivement » en position de fournir un jugement fondé concernant de la quantité d'information que le sujet sait avoir eu à sa disposition, ou encore par la provenance de cette information. $»^{5}$ 
utôt que de parler d'« erreurs » ou de «biais » dans le traitement de l'information sur autrui, une conception «socio-cognitive» des jugements nous permet de mieux comprendre comment "l'organisation des personnes, de leurs relations, répond également à la nécessité de mettre de l'ordre dans la structure sociale, dans ses règles d'organisation... $»^{6}$. La structure de la personnalité d'un individu se situe bien en effet dans la sphère cognitive de celui qui juge que dans le sujet dont on observe le comportement ${ }^{7}$. La communication est ici déterminée par un ensemble de compétences qui structurent les comportements du couple évaluateur/évalué, c'est-à-dire qui leur permettent de parler et d'agir en appliquant correctement l'ensemble des critères d'évaluation propres à leur culture en matière de vérité/justice/efficience/beauté/ déviance/expression non-verbale... Une défaillance dans l'application de ces règles de communication, non-verbales et verbales, est immédiatement considérée comme une incohérence de l'ensemble de la situation, ceci se traduit alors par un rejet (sanction) de l'évalué et une prise de position plus forte de l'évaluateur. Le juge évacuerait l'incohérence en la rationalisant sur un mode plutôt dévalorisant pour l'évalué. Dans cette optique la connaissance implicite des références en matière de norme attitudinale apparaît comme une véritable compétence sociale (ou méta-connaissance) qui permettrait de mieux se conformer aux attentes des évaluateurs sociaux, qu'ils soient juges, recruteurs ou enseignants. Si c'est une connaissance partagée qui rend possible les inférences et qui opère au niveau des significations, c'est aussi l'image du soi social, défini par le statut, qui confère à l'évaluateur social sa " capacité » à juger. Cette image ou «face» telle que l'a définit Erwing Goffman ne saurait être «jouée » de la même façon par tous les individus. Dans une situation de jugement social, les écarts révélés au travers des comportements non-verbaux seraient à la fois l'expression initiale et la justification finale de l'interaction.

\section{BIBLIOGRAPHIE}

ALDROVANDI M., GRYSELIER M., « Théories implicites de la personnalité professionnelle » in Psychologie Française, juillet 1986

ASCH S., Social Psychology, Englewood Cliffs, N.J. Prentice-Hall. 1952.

BARRIER G.. La communication non-verbale, LSF, Paris, 1996.

BEAUFILS B., Paicheler H.. Ravaud J-F., « Personnalisation et stigmatisations sociales », in Perspectives cognitives et conduites sociales, 1, Cousset-Delval, 1989.

BEAUVOIS J-L.. La psychologie quotidienne, PUF, Paris. 1984.

BROSSARD A. et COSNIER J., La communication non-verbale, Delachaux et Niestlé. 1984.

BURKE D., Ames A., Etherington R. \& Pietsch J.. « Effects of victim's and defendant's physical attractiveness on the perception of responsability in a ambigous domestic violence case ». Journal of Social Behavior and Personality. 1999. 
CASTELLOW W., " The effects of physical attractiveness and social desirability on judgements regarding a sexual harassment case ", Journal of Social Behavior and Personnality. 9(4), pp. 715-730. 1994.

CASTELLOW W., Wuensch K., Moore C, « Effects of physical attractiveness of the plaintiff and defendant in sexual harassment judgement ». Journal of Social Behavior and Personality. 5 (6), pp. 547-562. 1990. Chapman I... Chapman J., « Illusory correlation as an obstacle to the use of valid psychodiagnostic signs ", in Journal of abnormal Psychology, 1969. vol. 74.

COFFRE P., Les déterminants non-verbaux dans l'acte de vente. Thèse en sciences de gestion. Ecole Supérieure des Affaires de Grenoble, 1998. Darley J., « A hypothesis-confirming bias in labeling effects ", Journal of Personality anf Social Psychology. 43. pp. 20-33. 1983. De Gail M-A., Le psychologisme. un mode de communication " utile ", Thèse de doctorat, sous la direction d'Armand Mattelart. Rennes, 1994. Despret V., Naissance d'une théorie éthologiquc. Ed. Synthelabo, Paris. 1996.

DROUIN-LLANS A-M.. La communication non-verbale avant la lettre, L'Harmattan. Paris, 1995.

GOFFMAN E., Les rites d'interaction. Minuit. Paris. 1974. Hall F. T., Le langage silencieux. Seuil. Paris. 1986.

HUBBARD M., Lepper M., Ross L., « Perceverance in self-Perception Social Perception : Biaised Attibutional Process in the Debriefing Parading », Journal of Personnality and Social Psychologie, 1975, vol 32.

JAKOBI J-M. et MASSE L., « Le regard dans le discours politique » in Psychologie Française, PUG, déc 1999 , tome $44-\mathrm{N}^{\circ} 4$.

LE POULTIER F., Recherches sur les pratiques évaluatives en travail social, PUG, Grenoble, 1990.

LEYENS J-P., Sommes-nous tous des psychologues ?, Mardaga, Bruxelles, 1986.

LYR G., Face au public : parler de tout son corps, Ed. d'Organisation, Paris. 1999.

MONTEIL J-M., Eduquer et Former PUG, Grenoble, 1989.

MOSCOVICI S., « De la science au sens commun » in Psychologie sociale sous

la direction de Serge Moscovici, PUF, Paris, 1984.

NISBETT R., « Verbal reports about causal influence on social judgements », Journal of Personality anfSocial Psychology, 1977. 35, pp. 613-624. Paicheler H., « L'epistemologie du sens commun, de la perception à la connaissance de l'autre » in Psychologie sociale sous la direction de Serge Moscovici, PUF, Paris, 1984.

ROGE B., Différenciation sexuelle et communications non-verbales: Approche développement aie, Thèse de Doctorat d'état, Paris VIII, 1992. Schadron G. et Yzerbit V.. « L'impact des informations au-delà de leur contenu : Application de l'approche de la jugeabilité sociale aux stéréotypes », Perspectives cognitives et conduites sociales, $\mathrm{n}^{\circ}$ 4, Delachaux etNiestlé, 1995.

ZIMMERMANN D., La sélection non-verbale à l'école, ESF. Paris. 1982.

\section{NOTES}

1. Monteil Jean-Marc Eduquer et former, Grenoble. PUG, 1989.

2. Burke Donald, Ames Ashey, Etherington Rosalie \& Pietseh Jean, «Effect of victim's and defendant's physical attractiveness on the perception of responsability in a ambigous domestic violence case ", Journal of Social Behavior and Personality, 1999. 
3. Marie-Agnès de Gail, Le psychologisme, un mode de communication "utile ", Thèse de doctorat, sous la direction d'Armand Mattclart, Rennes, 1994

4. Georges Schadron et Vincent Y/erbit, "L'impact des informations au-delà de leur contenu : Application de l'approche de la jugeabilité sociale aux stéréotypes». Perspectives cognitives et conduites sociales, $\mathrm{n}^{\circ} 4$, Cousset-Delval, 1995.

5. Georges Schadron, Vincent Yzerbit, «L'impact des informations au-dela de leur contenu: application de l'approche de la jugeabilité sociale aux stéréotypes" in Jugeabilitè sociale et stéréotype sous la direction de G. Schadron.

6. Henri Paicheler, «L'epistemologie du sens commun : de la perception à la connaissance de l'autre » in Psychologie sociale sous la direction de S. Moscovici, Paris PUF, 1984.

7. Jean-Marc Monteil op. cit.

\section{AUTEURS}

\section{NICOLAS GUÉGUEN}

Laboratoire Gresico Université de Bretagne Sud IUTde Vannes 